\title{
Design of an Ultra-Wideband Waveform Shaping Network Using an Analog Microwave Differentiator Array fed by a Gaussian Pulse Signal
}

\author{
Chih-Yung Tsai* and Shyh-Kang Jeng \\ Graduate Institute of Communication Engineering \\ National Taiwan University \\ Taipei, Taiwan, ROC \\ Email: d89006@ew.ee.ntu.edu.tw \\ skjeng@ew.ee.ntu.edu.tw
}

\begin{abstract}
We propose a new method for the generation of ultra-wideband (UWB) signals which use as much bandwidth as possible to maximize the emitted power. This method is different from the filtering approach which may be simple to generate UWB signals but lacks the capability of precisely shape and position UWB signal energy in the frequency spectrum. In our approach, we feed a Gaussian pulse into an analog microwave differentiator array, then, the outputs are combined by a suitably designed power combiner after appropriate signal amplifications and/or attenuations. Because the $n$-th order derivative of a Gaussian pulse will move the energy concentration part to higher frequency band as $n$ increases, we use this property to design the desired pulse whose energy is distributed at the desired frequency band to meet some specific regularity in UWB applications.
\end{abstract}

\section{Introduction}

Unlike the conventional narrow-band communication systems, it is desirable for ultrawideband (UWB) communication systems to employ very short pulses with different modulations that result in extremely wideband spectra for high data-rate links. This kind of UWB signals spreads the energy as widely in frequency as possible to minimize the power spectral density (PSD) and hence reduces the potential for interference with other systems. On the other hand, these ultra-wideband signals result in extremely short pulses in time domain which possess excellent multi-path immunity. In recent years, there are researches which indicate that the best pulse shapes for different requirements are different $[1,2]$, and the signal transmitting characteristics display significant reliance on pulse shapes for many reasons such as PSD, ease of pulse shape generation, peak-toaverage power ratios, etc.. And some methods have been proposed to design the optimal pulse shapes for UWB communications $[3,4,5]$. The Gaussian pulse possesses some nice properties such that it becomes a common choice for UWB applications. The design of Gaussian pulse generator has been proposed in [6,7] and has already been available from some electronic vendors.

In UWB applications, the signal's equivalent isotropically radiated power (EIRP) must conform to the regulation defined by the Federal Communication Commission (FCC) [8]. Due to the reason that the closer the signal PSD conforms to the area under the FCC PSD mask the more power the signal can be made to transmit. If all available spectra from 3.1 to $10.6 \mathrm{GHz}$ were perfectly filled with the maximum allowed signal PSD without filtering, the signal power efficiency will be the maximum. On the other hand, because the $n$-th order derivative of a Gaussian pulse will move the energy concentration part to higher frequency band as $n$ increases. So, in our method, we take the FCC PSD mask as the 
target function, and choose the Gaussian pulse and the pulses of its higher order derivatives as a set of basis functions to synthesize the best pulse to meet the maximum signal power efficiency requirement. The schematic diagram of our waveform shaping network is shown in Fig. 1.

In the researches of pulse shape optimization and pulse shape design as seen in $[1,3,4,5]$ have proposed new theory or method from mathematical viewpoint without practical circuit implementation. In our research, at first, we try to find out a better combination of the basis functions using some mathematical method with consideration about tradeoff between the performance and the implementation of the circuit complexity. Then, we try to design an analog microwave differentiator to perform differentiation on the input Gaussian pulse. The third step is to design a suitable power combiner to combine the output signals from the differentiators.

\section{Analysis and Design}

Step 1: Apply the least square method (LSM) to design the waveform shaping network:

Given a desired PSD mask $F(\omega)$, the approximation of $F(\omega)$ is $Y(\omega)=W(\omega) H(\omega)$, where $H(\omega)=\sum_{i=0}^{n} a_{i}(j \omega)^{i}$ which represents an analog microwave differentiator array, and $W_{0}(\omega)=F . T .\left\{A e^{-\left(\frac{t-t_{c}}{\tau}\right)^{2}}\right\}$ is the Fourier transform of the Gaussian pulse. Then, we solve the problem $\min \left\|F(\omega)-W_{0}(\omega) \sum_{i=0}^{n} a_{i}(j \omega)^{i}\right\|^{2}$ to find a suitable $a_{i}$ to synthesize the pulse in frequency domain. The numerical results are shown in Fig. 2 and Fig. 3 which represents the best signal PSD without considering the implementation complexity and the one less complicate but good enough which we have adopted to fulfill our design, respectively.

Step 2: Design a $2^{\text {nd }}$-order differentiator unit:

In this step, we apply a finite difference to approximate the differentiation operation, and use transmission line (T.L.) sections and short stubs to fulfill the differentiator circuit. The concept is very straightforward for the approximation $(\Delta t) f^{\prime}(t) \approx f(t+\Delta t)-f(t)$ and the schematic diagram is shown in Fig. 4. Besides, the RC differentiator from textbook of electronics [9] is also adopted. Although it has a restriction that the differentiation is valid only for $\omega R C \ll 1$ which severely decreases the amplitude magnitude of the output signal, we found that the performance is better when combine it with the T.L. one than cascade two T.L. sections. Thus we cascade a T.L. $1^{\text {st }}$-order differentiator with an $\mathrm{RC}$ one to form a $2^{\text {nd }}$-order differentiator unit (SODU), and use it to assemble the differentiator array as indicated in Fig.1. In Fig. 5, we show the first 6 differentiated pulse outputs of our differentiator circuits with the Gaussian pulse input. The results were simulated using the Advanced Design System ${ }^{\circledR}$ of Agilent Technologies. From the results with regardless of the polarities, we can confirm our differentiators work well with acceptable accuracy from $1^{\text {st }}$ to $3^{\text {rd }}$ order differentiations. Although there are slight distortions for the $4^{\text {th }}$ and $5^{\text {th }}$ order differentiations, the pulse shapes are very close 
to the exact ones. The ringing effect for the $6^{\text {th }}$ differentiation may cause some problems. Another possible problem is that our differentiators have increased the pulse width slightly which may indicate that the bandwidth of our circuit is not sufficient to meet our specification.

Step 3: Deal with the problem of signal combination:

In this step, we choose a 3-way unequal-split Wilkinson power divider/combiner as the candidate. We normalize the largest $a_{i}$ to 1 to obtain the power combination ratio and apply the systematic methods in $[10,11]$ to design the one which meet our requirements. Some delay lines between the output ports of our differentiator array and the input ports of the power combiner are required to synchronize the output signals before power combination. Due to the results we have obtained are not satisfied yet, we don't present them in this paper.

\section{Conclusions}

We have proposed a new method to design a better pulse shape for UWB applications but also propose a practical circuit architecture to generate the optimal pulse which we have obtained from optimization method. If it is combined with the circuit of Gaussian pulse generator such as that in [6], then, only a square pulse input is required. On the other hand, the circuit of our design and [6] are all planar structure which can be integrated as a single circuit unit. This method is flexible and can be applied to other radio systems with the same design procedure and circuit architecture. Finally, the differentiators designed in this research can be a very useful tool to shape other signals.

\section{References}

[1] David M. Pozar, "Waveform Optimizations for Ultrawideband Radio Systems," IEEE Transactions on Antennas and Propagation, Vol. 51, No. 9, Sep., 2003

[2] X. Chen and S. Kiaei, "Monocycle shapes for ultra wideband system", IEEE ISCAS 2002., Vol.1, 26-29 May 2002

[3] C. A. Corral, S. Sibecas, S. Emami, and G. Stratis, "Pulse spectrum optimization for ultra-wideband communication," IEEE Conference on UWBST 2002, Digest of Papers, pp.31-35, 21-23 May 2002

[4] B. Parr, B. Cho, K. Wallace, and Z. Ding, "A Novel Ultra-Wideband Pulse Design Algorithm", IEEE Communication Letters, Vol.7, No.5, May,2003

[5] R. S. Dilmaghani, M. Ghavami, B. Allen, and H. Aghvami, "Novel UWB pulse shaping using prolate spheroidal wave functions", IEEE Proceedings on PIMRC 2003, Vol.1, Sep. 2003

[6] J. Han and C. Nguyen, "A New Ultra-Wideband, Ultra-short Monocycle Pulse Generator With Reduced Ringing", IEEE Microwave and Wireless Components Letters, Vol.12, No.6, Jun. 2002

[7] H. Kim, D. Park, and Y. Joo, "Design of CMOS Scholtz's Monocycle Pulse Generator", , IEEE Conference on UWBST 2003

[8] Federal Communications Commission, First report and order, revision of part 15 of the commission's rule regarding ultra wideband transmission systems, FCC 02-48, April 22, 2002.

[9] Horowitz and Hill, The Art of Electronics, Cambridge Press, $2^{\text {nd }}$ Edition, 1998.

[10] D. M. Pozar, Microwave Engineering, John Willy \& Sons Inc., $2^{\text {nd }}$ Edition, 1998. 
[11] L. I. Parad and R. L. Moynihan, "Split-Tee Power divider," IEEE Trans. on Microwave Theory and Techniques, Vol. 13, No. 1, Jan. 1965

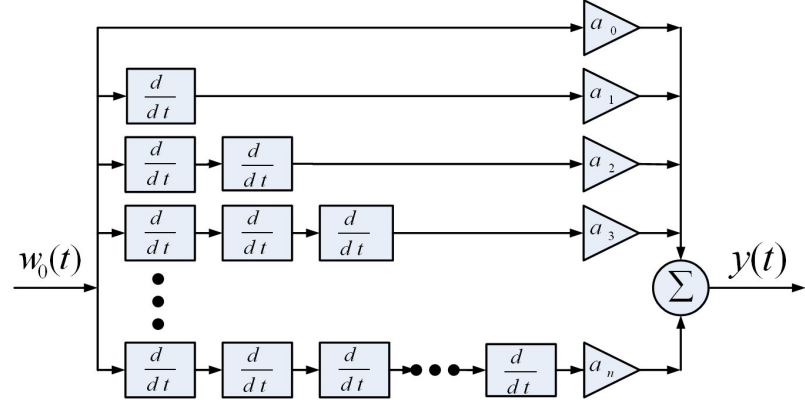

Fig. 1: The schematic diagram of our network.

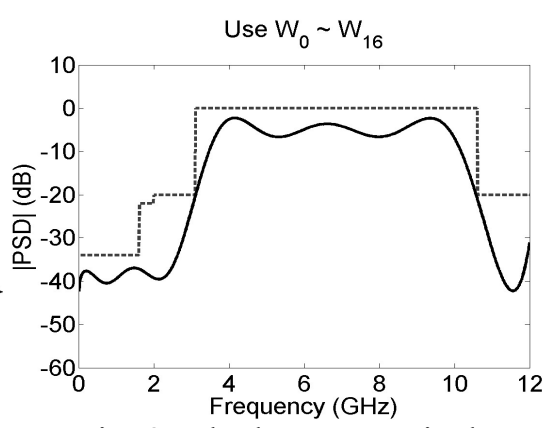

Fig. 2: The best numerical result.
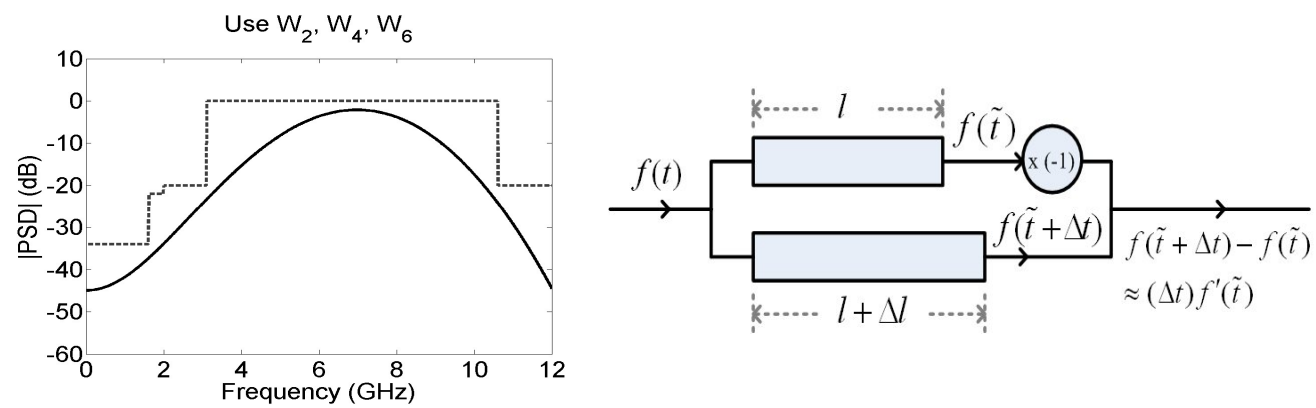

Fig. 3: The result to implement our design. Fig. 4: The $1^{\text {st }}$ order differentiator using T.L.

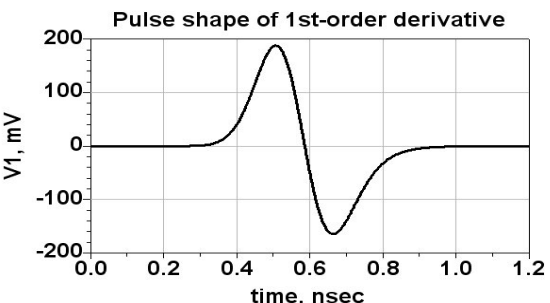

(a)

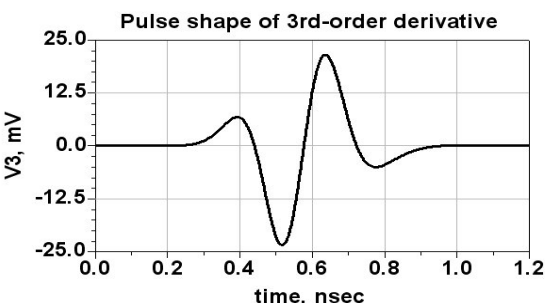

(c)

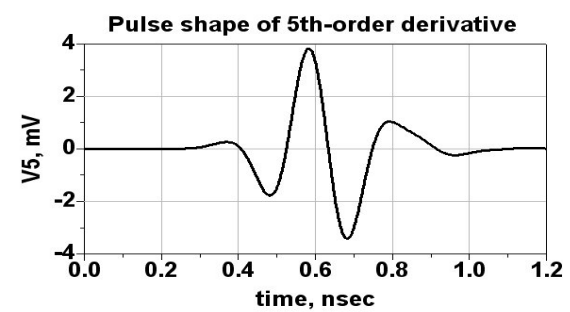

(e)

(f) (b)

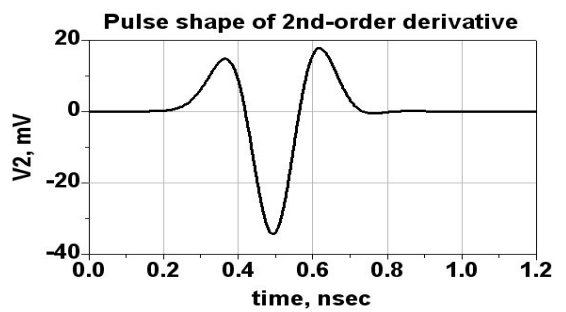

(d)
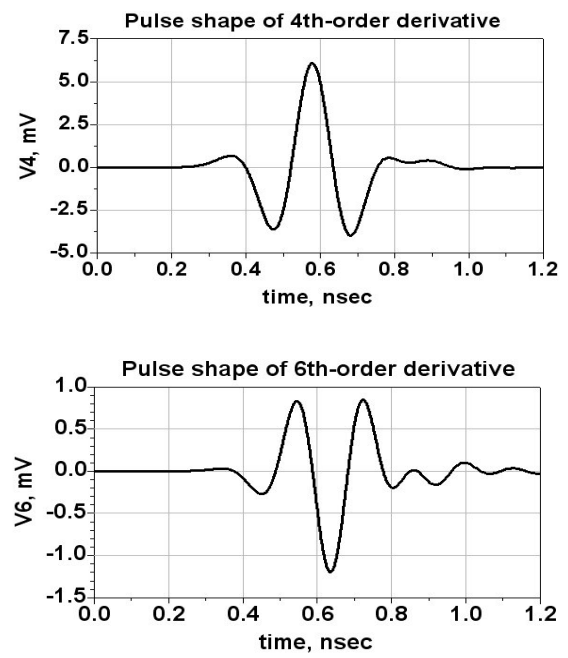

Fig. 5: From (a) to (f): $1^{\text {st }}$ to $6^{\text {th }}$ order differentiated pulse outputs of our differentiator circuits with the Gaussian pulse input. 